University of Nebraska - Lincoln

DigitalCommons@University of Nebraska - Lincoln

February 2007

\title{
PHOTOMETRY OF TYPE II CEPHEID CANDIDATES FROM THE ROTSE-I DEMONSTRATION PROJECT
}

\author{
Edward G. Schmidt \\ University of Nebraska-Lincoln, eschmidt1@unl.edu \\ Shawn Langan \\ University of Nebraska - Lincoln \\ Danielle Rogalla \\ University of Nebraska - Lincoln \\ Lauren Thacker-Lynn \\ University of Nebraska - Lincoln
}

Follow this and additional works at: https://digitalcommons.unl.edu/physicsschmidt

Part of the Physics Commons

Schmidt, Edward G.; Langan, Shawn; Rogalla, Danielle; and Thacker-Lynn, Lauren, "PHOTOMETRY OF TYPE II CEPHEID CANDIDATES FROM THE ROTSE-I DEMONSTRATION PROJECT" (2007). Edward Schmidt Publications. 34.

https://digitalcommons.unl.edu/physicsschmidt/34

This Article is brought to you for free and open access by the Research Papers in Physics and Astronomy at DigitalCommons@University of Nebraska - Lincoln. It has been accepted for inclusion in Edward Schmidt Publications by an authorized administrator of DigitalCommons@University of Nebraska - Lincoln. 


\title{
PHOTOMETRY OF TYPE II CEPHEID CANDIDATES FROM THE ROTSE-I DEMONSTRATION PROJECT
}

\author{
Edward G. Schmidt, Shawn Langan, Danielle Rogalla, and Lauren Thacker-Lynn \\ Department of Physics and Astronomy, University of Nebraska, Lincoln, NE, USA; eschmidt1@unl.edu, \\ nuaur@hotmail.com,drogall1@bigred.unl.edu, lologur1329@aol.com \\ Received 2006 October 13; accepted 2006 October 20
}

\begin{abstract}
We have obtained VR photometry of 205 Cepheid variable star candidates that were discovered in a small section of the Northern Sky Variability Survey. Given their locations and apparent magnitudes, any stars in this sample that are Cepheids are very likely to be type II Cepheids. On the basis of the regularity of variation, revised periods, lightcurve morphology, slope of the color-magnitude relation, and color, we have identified 97 probable Cepheids and 17 possible Cepheids. While some of the photometric properties, particularly the colors, are consistent with the identification of these stars as Cepheids, the amplitudes are small compared to known type II Cepheids and the period distribution does not match that of known type II Cepheids.
\end{abstract}

Key words: Cepheids — stars: Population II

Online material: machine-readable tables

\section{INTRODUCTION}

Type II Cepheid variable stars offer great, but largely unrealized, potential to contribute to various areas of astrophysics. Just as the classical Cepheids were important for testing post-mainsequence stellar evolution theory (see Chiosi [1990] for a summary and references to earlier work), type II Cepheids can provide useful constraints on post-horizontal branch stellar evolution models (Gingold 1985). The properties of type II Cepheids indicate that while some are members of the Galactic halo, many are associated with the thick disk. Thus, they are potential probes of the structure, stellar content, and history of the halo and thick disk. The presence of hydrogen emission and large velocity differentials in the spectra of some type II Cepheids is of interest in connection with stellar pulsation and atmospheric dynamics (Schmidt et al. 2003, 2004b). Finally, the type II Cepheids and the closely related RV Tau stars are of interest in connection with the possible presence of chaos in pulsating stars (see Kovacs \& Buchler 1988 and references therein).

Unfortunately, the application of type II Cepheids to these interesting areas is seriously limited by the available sample. The General Catalog of Variable Stars (GCVS; with corrections and additions from its online version) ${ }^{1}$ only lists 183 type II Cepheids (types CWA, CWB, and CW) and 173 Cepheids of uncertain type (CEP). Most of the latter are likely to be classical Cepheids. Harris' (1985) catalog of type II Cepheids lists 152 selected from the GCVS on the basis of location and an additional 56 selected on other grounds. Thus, the total number of known field type II Cepheids is little more than 200. In addition, there are about 60 Cepheids known in globular clusters (Clement et al. 2001). The limited number is further aggravated by the wide range in properties of the type II Cepheids; any reasonably homogeneous subset will be quite small.

Based on the known globular cluster Cepheids, Wallerstein (2002) estimated that there should be about 6000 field Cepheids in the halo. This suggests that a large majority of type II Cepheids remain undiscovered.

In recent years large-area sky surveys have detected literally thousands of new, relatively bright variables. These include the

\footnotetext{
${ }^{1}$ VizieR Online Data Catalog, II/214A (P. N. Kholopov et al., 1996).
}

All Sky Automated Survey (Pojmanski et al. 2005 and references therein), the Hungarian Automated Telescope survey (Bakos et al. 2002; Hartman et al. 2004), and the Northern Sky Variability Survey (NSVS; Wozniak et al. 2004), which was derived from Robotic Optical Transient Search Experiment I (ROTSE-I) data. Thus, there is now a fresh opportunity to identify the undiscovered type II Cepheids. The present paper reports observations of Cepheid candidates that were found by Akerlof et al. (2000) in a portion of the NSVS. Future studies will expand the sample to include stars found in the other parts of the NSVS and in other surveys.

\section{THE SAMPLE OF STARS}

To demonstrate the potential of the ROTSE-I data set for discovering variable stars, Akerlof et al. (2000) searched a subset of the data encompassing three months of observations and covering about $5 \%$ of the sky. They identified 1781 periodic variables brighter than $V=15.5,90 \%$ of which were new discoveries. The light-curve shapes were used to classify the stars as various types of pulsators or as eclipsing or contact binaries.

In spite of the large amount of data available in the NSVS, further photometry is needed to identify bona fide type II Cepheids and to explore their properties. Because the survey observations were unfiltered, the magnitudes cannot be placed accurately on a standard magnitude system and color information is lacking. The internal errors in the NSVS data increase from about $0.015 \mathrm{mag}$ at $10 \mathrm{mag}$ to about $0.07 \mathrm{mag}$ at $14 \mathrm{mag}$, a range that encompasses most of the stars of interest here. Even with a large number of observations, this can compromise the determination of the form of light curves for small amplitude variables. Intrinsic scatter in light curves has proven to be a useful diagnostic of type for some stars (Schmidt et al. 2004a), and this too is compromised by observational scatter. Finally, further photometry will provide information on the long-term behavior of the variables. This is important to distinguish between Cepheids and similar stars, such as yellow semiregular variables.

We selected 205 Cepheid candidates from the Akerlof et al. (2000) list. This includes all of the stars that they classified as pulsating variables with periods in the range from 0.98 to 190 days. The extension to longer periods than usually associated with 
TABLE 1

Program Stars

\begin{tabular}{|c|c|c|c|c|c|c|c|}
\hline $\begin{array}{l}\text { No. } \\
\text { (1) }\end{array}$ & $\begin{array}{l}\text { ROTSE-I Designation } \\
\text { (2) }\end{array}$ & $\begin{array}{c}\text { GCVS Name } \\
\text { (3) }\end{array}$ & $\begin{array}{l}\text { Period }^{\mathrm{a}} \\
\quad(4)\end{array}$ & $\begin{array}{c}\langle m\rangle^{\mathrm{a}} \\
(5)\end{array}$ & $\begin{array}{c}\Delta m^{\mathrm{a}} \\
(6)\end{array}$ & $\begin{array}{c}N_{\text {obs }} \\
\text { (Behlen) } \\
(7)\end{array}$ & $\begin{array}{c}N_{\text {obs }} \\
\text { (NSVS) } \\
(8)\end{array}$ \\
\hline R001 & J190813.10+382958.8 & $\ldots$ & 0.983338 & 12.10 & 0.376 & 30 & 33 \\
\hline 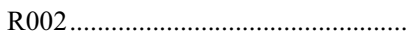 & $\mathrm{J} 175945.41+313519.3$ & $\ldots$ & 1.016348 & 13.50 & 0.281 & 18 & 494 \\
\hline R003 & $\mathrm{J} 174252.68+230104.4$ & $\ldots$ & 1.02233303 & 11.23 & 0.301 & 20 & 243 \\
\hline R004 & $\mathrm{J} 173317.20+255526.5$ & $\ldots$ & 1.02267396 & 12.45 & 0.163 & 20 & 141 \\
\hline R005 & $\mathrm{J} 184211.03+390735.3$ & $\ldots$ & 1.02331805 & 11.85 & 0.173 & 36 & 277 \\
\hline
\end{tabular}

Nоте.-Table 1 is published in its entirety in the electronic edition of the Astronomical Journal. A portion is shown here for guidance regarding its form and content.

${ }^{a}$ From Akerlof et al. 2000.

Cepheids was intended to ensure that we included the longest period Cepheids, RV Tau stars, and stars with erroneously long periods.

In Table 1 we have listed stars classified by Akerlof et al. (2000) as Cepheids (their class C, 201 stars) followed by four that they classified as RR Lyrae (RRAB, RRC). In each group, stars are listed in order of the periods given by Akerlof et al. Column (1) gives a running number for use in this paper, and column (2) contains the designation of the star from Akerlof et al. (2000). Column (3) gives the variable-star names for objects included in the GCVS. In columns (4), (5), and (6) we list the periods, mean magnitudes, and amplitudes given by Akerlof et al. The last two columns are discussed below.

Akerlof et al. (2000) studied a strip of the sky that extended from a Galactic latitude of $b \approx 10^{\circ}$ to the north Galactic pole. Nearly all of the stars in the selected sample have ROTSE magnitudes between 10 and 14. A classical Cepheid fainter than
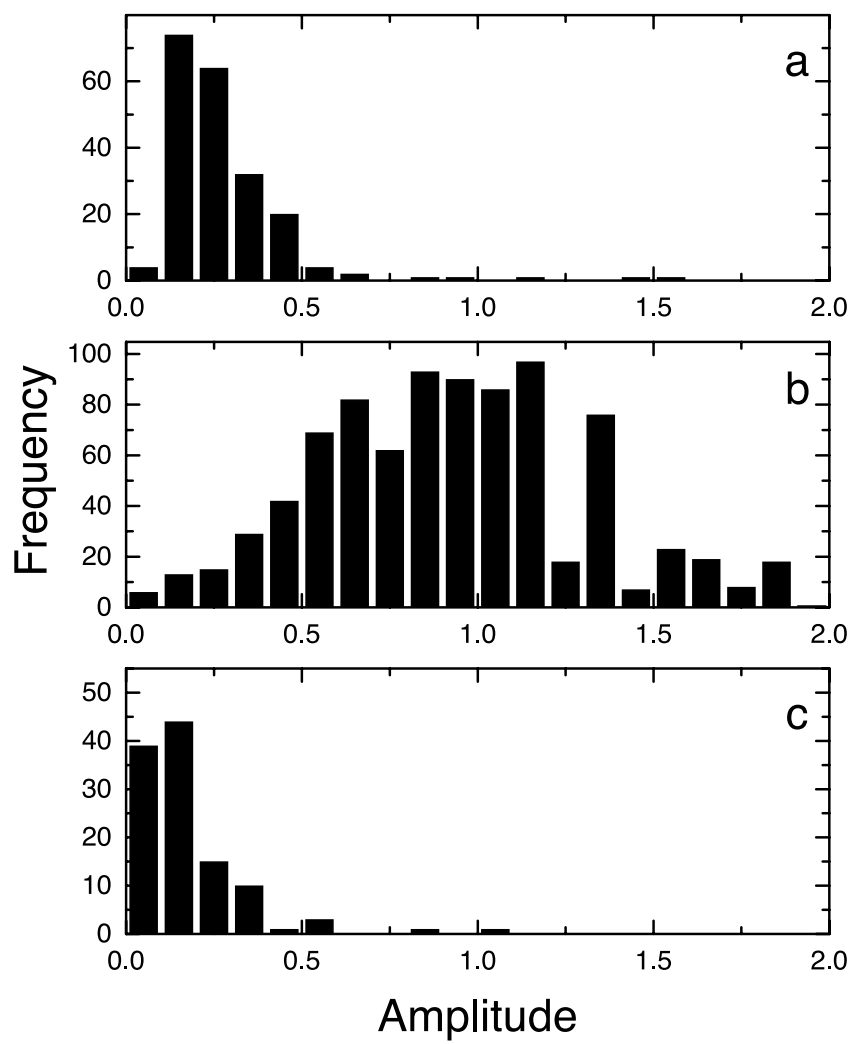

FIG. 1.-Amplitude distributions with period: $(a)$ amplitudes from Akerlof et al. (2000) for our full sample; $(b)$ amplitudes from the GCVS for stars classified as Cepheids; (c) Vamplitudes for Cepheid candidates (both status A and status B in col. [12] in Table 3) from the present study.
10 mag at a latitude above $10^{\circ}$ would be at least $350 \mathrm{pc}$ from the Galactic plane. Since the scale height of classical Cepheids is about 70 pc (Harris 1985) we conclude that virtually all of the Cepheids in the Akerlof et al. (2000) sample are type II Cepheids.

Figure $1 a$ displays the frequency distribution of the amplitudes from Akerlof et al. (2000) (col. [6] of Table 1) of the stars in our selected sample. Figure $1 b$ shows the distribution of amplitudes from the GCVS for stars that are classified as some type of Cepheid (GCVS classifications of CEP, DCEP, CW, CWA, and $\mathrm{CWB}$ ). Even allowing for the fact that the amplitudes from Akerlof et al. (2000) are based on unfiltered magnitudes and those from the GCVS are based on several different filter bands, the difference between the two histograms is striking. This would be equally true if we restricted Figure $1 b$ to stars thought to be type II Cepheids or if we expanded it to include all stars in the Cepheid period range from the GCVS that appear to be pulsating variables. Clearly, the survey of Akerlof et al. (2000) has uncovered a population of low-amplitude variable stars that is largely missing from the GCVS. One of the goals of this project is to determine which of these stars are Cepheids and to shed light on the nature of those that are not Cepheids.

\section{THE DATA}

\subsection{The Observations}

The new observations reported here consist of 3383 VR photometric observations made between 2003 September and 2006 May (JD 2,452,899-2,453,888) at Behlen Observatory. The instrumentation and the procedures for collecting and processing the data were described by Schmidt et al. (2004a and references therein) and the reader is referred to that publication for details. The only deviation was the use of the same detector, the Princeton Instruments VersArray:1300B CCD Camera, throughout this project. The number of observations for each star is listed in column (7) of Table 1, while individual photometric observations are presented in Table 2.

TABLE 2

Photometric Data

\begin{tabular}{|c|c|c|c|}
\hline Star & HJD - 2,400,000 & V & $V-R$ \\
\hline R001 . . . . . . . . . . . . & $52,905.761$ & 14.038 & 1.420 \\
\hline R001 .............................. & $52,913.677$ & 13.938 & 1.383 \\
\hline 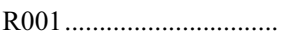 & $52,913.694$ & 13.926 & 1.389 \\
\hline 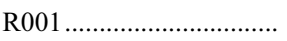 & $52,913.738$ & 13.925 & 1.389 \\
\hline 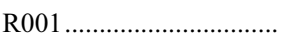 & $52,914.672$ & 13.939 & 1.385 \\
\hline
\end{tabular}

Noте.-Table 2 is published in its entirety in the electronic edition of the Astronomical Journal. A portion is shown here for guidance regarding its form and content. 
TABLE 3

Light-Curve Parameters

\begin{tabular}{|c|c|c|c|c|c|c|c|c|c|c|c|}
\hline $\begin{array}{l}\text { No. }^{a} \\
(1)\end{array}$ & $\begin{array}{l}\text { Period }^{b} \\
\text { (2) }\end{array}$ & $\begin{array}{l}\sigma_{P} \\
\text { (3) }\end{array}$ & $\begin{array}{l}\langle V\rangle \\
(4)\end{array}$ & $\begin{array}{l}\sigma_{V} \\
(5)\end{array}$ & $\begin{array}{c}\langle V-R\rangle \\
\quad(6)\end{array}$ & $\begin{array}{c}\sigma_{V-R} \\
\text { (7) }\end{array}$ & $\begin{array}{l}\Delta V \\
(8)\end{array}$ & $\begin{array}{c}S \\
(9)\end{array}$ & $\begin{array}{c}\sigma_{S} \\
(10)\end{array}$ & $\begin{array}{l}\text { Notes }^{\mathrm{c}} \\
(11)\end{array}$ & $\begin{array}{c}\text { Status }^{\mathrm{d}} \\
(12)\end{array}$ \\
\hline 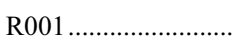 & (57) & $\ldots$ & (13.9) & $\ldots$ & (1.4) & $\ldots$ & 0.38 & 0.11 & 0.04 & $\mathrm{RE}$ & \\
\hline 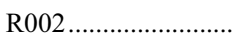 & (320) & $\ldots$ & $(14.0)$ & $\ldots$ & $(1.0)$ & $\ldots$ & $(0.29)$ & 0.08 & 0.03 & $\mathrm{RE}$ & B \\
\hline 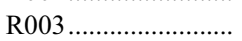 & $\ldots$ & $\ldots$ & (13.7) & $\ldots$ & (1.9) & $\ldots$ & $(0.44)$ & 0.33 & 0.04 & IR, RE & \\
\hline 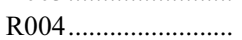 & (78) & $\ldots$ & (13.3) & $\ldots$ & $(1.1)$ & $\ldots$ & $(0.3)$ & 0.15 & 0.02 & IR, RE & \\
\hline 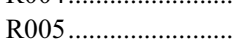 & $\ldots$ & $\cdots$ & $(12.5)$ & $\begin{array}{l}\cdots \\
\cdots\end{array}$ & (1.0) & $\begin{array}{l}\cdots \\
\cdots\end{array}$ & $(0.2)$ & 0.14 & 0.03 & IR, RE & \\
\hline
\end{tabular}

Notes.-Table 3 is published in its entirety in the electronic edition of the Astronomical Journal. A portion is shown here for guidance regarding its form and content.

${ }^{\text {a }}$ Notes on individual stars. R007: Only one photometric night, so the zero point of the magnitude and color are uncertain. R008: Excess scatter suggests that this star exhibits the Blazhko effect or multiperiodicity. R069: The Akerlof period was used for this star since a period could not be determined from the Behlen data. R074: This star appears to have undergone a sudden period change during the interval covered by the NSVS data. By the epoch of the Behlen data the period had reverted to the original value. The period listed in the table is valid for the NSVS data prior to JD 2,451,320 and the Behlen data. R092: The adjusted period satisfies both the first season of the NSVS data (JD 2,451,274-2,451,322) and the Behlen data. However, the amplitude of the former is much larger. No well-defined period could be found for the second season of the NSVS data (JD 2,451,512-2,451,633). R122: We are unable to find a period that satisfies all of the data. However, there is some indication of RV Tau behavior with a double period of about 83 days in the NSVS data. R137: The NSVS data only covers part of the cycle of this star. The cited period fits both the Behlen data and the NSVS data but must be regarded as tentative.

b The period was determined using only the present photometry for the following stars: R032, R034, R038, R043, R053, R063, R070, R080, R094, R096, R099, R106, R113, R131, R139, R154, R155, R157, R160, R161, R165, R173, R176, R177, R179, and R188. When a well-defined period could not be found but a characteristic timescale could be discerned, the timescale is given in parentheses.

${ }^{\mathrm{c}}$ The codes indicate reasons for rejecting the star as a Cepheid candidate: $\mathrm{BL}$, too blue to be a Cepheid; CO, constant star; CV, light curve indicates a cataclysmic variable; EC, light curve indicates an eclipsing binary; IR, irregularity of the period or light curve eliminates the star as a Cepheid; RE, too red to be a Cepheid; SL, the slope of the color-magnitude relation falls outside of the expected range for a Cepheid; SP, period too short for a Cepheid.

d "A" signifies a probable Cepheid and "B" a possible Cepheid. A blank entry indicates that the star was rejected as discussed in the text.

In the analysis below, we used both our new photometry and the magnitudes from the first public data release of the NSVS (Wozniak et al. 2004). These latter observations span a year's interval from 1999 April through 2000 March (JD 2,451,270$2,451,630)$. The number of NSVS observations for each star is listed in column (8) of Table 1. While the NSVS magnitudes were adjusted to the $V$ system on the average, the broad response band produces large magnitude shifts for stars of different colors. In addition, some of the stars in our sample show long-term variations in mean brightness. To roughly account for these factors, we added an offset to the NSVS magnitudes for each star to bring them to our $V$ scale. These corrections fell between -0.1 and 2.1 and were well correlated with the $\langle V-R\rangle$ color. While this matching of the two data sets is, at best, a very rough approximation, it is adequate to allow us to use all of the data in determining periods and ascertaining the long-term behavior of the stars.

\subsection{Photometric Properties}

Periods were determined for the program stars using the combined set of the NSVS data and our data. A first estimate was found with the data-compensated discrete Fourier transform method of Ferraz-Mello (1981). The light curve was then plotted and the period adjusted to optimally match the maxima and minima of the two data sets. An upper limit on the uncertainty of the period was determined by increasing and decreasing the period until an unacceptable mismatch resulted. The adopted periods are given in column (2) of Table 3, and the uncertainties are listed in column (3) in units of the last digit of the tabulated period. Since Akerlof et al. (2000) used data spanning a relatively short interval, it is not surprising that many of our revised periods differ from theirs.

A serious effort was made to find a period for each star that satisfied both the NSVS data and the present data, even if it was not the best period for the individual data sets. For a few stars this was not possible. In those cases we have either listed a period based only on our data (indicated by footnote $b$ in the table) or left column (2) blank if our data were not adequate to determine a reasonably secure period.

A well-defined period could not be found for some stars due to obvious irregularity. When a characteristic timescale could be discerned, we have given it in parentheses in column (2). If this was not possible, the entry is left blank.

Intensity mean $V$ magnitudes and $V-R$ colors for the stars are listed in columns (4) and (6) of Table 3 . When a period is given, these were calculated by converting the $V$ and $R$ magnitudes to intensities and using trapezoidal integration. The estimated uncertainties are listed in columns (5) and (7) in units of $0.01 \mathrm{mag}$. These were determined by combining in quadrature the standard errors of the zero points of the comparison stars, the uncertainty introduced by scatter in the light curves, and the uncertainty introduced by incomplete phase coverage. The light-curve scatter was found by fitting a low-order Fourier series and determining the scatter about it. The uncertainty introduced by phase coverage was estimated with a Monte Carlo simulation.

When no period is given for a star, columns (4) and (6) list arithmetic means of the measured magnitudes and colors and are enclosed in parentheses. In such cases no error is listed.

Column (8) of Table 3 gives the amplitude of the light curve in $V$. This was taken to be the amplitude of a low-order Fourier fit to the light curve when there was an adequate number of welldistributed points. In a few cases the fit did not represent the data near the maxima or minima, so the amplitude was increased to account for the deviation. When no good fit could be obtained, either due to the paucity of data, irregular behavior of the star, or the lack of a period, the value in column (8) is the range of measured $V$ magnitudes. Such cases are enclosed in parentheses.

In column (9) of Table 3, we list the slope, $S=\Delta(V-R) /$ $\Delta V$, of the color magnitude relation over the cycle for each star. The standard deviation of the slope is given in column (10). The final two columns of Table 3 contain notes concerning the status of each star as discussed below.

\section{ANALYSIS}

The goal of this project is to identify objects that are likely to be type II Cepheids. To accomplish this, we use the photometric properties to eliminate non-Cepheids from the sample.

Scatter is rather common in the light curves of type II Cepheids, and they often exhibit relatively large period changes (Schmidt et al. 2004a, 2005a, 2005b). However, those for which we either 

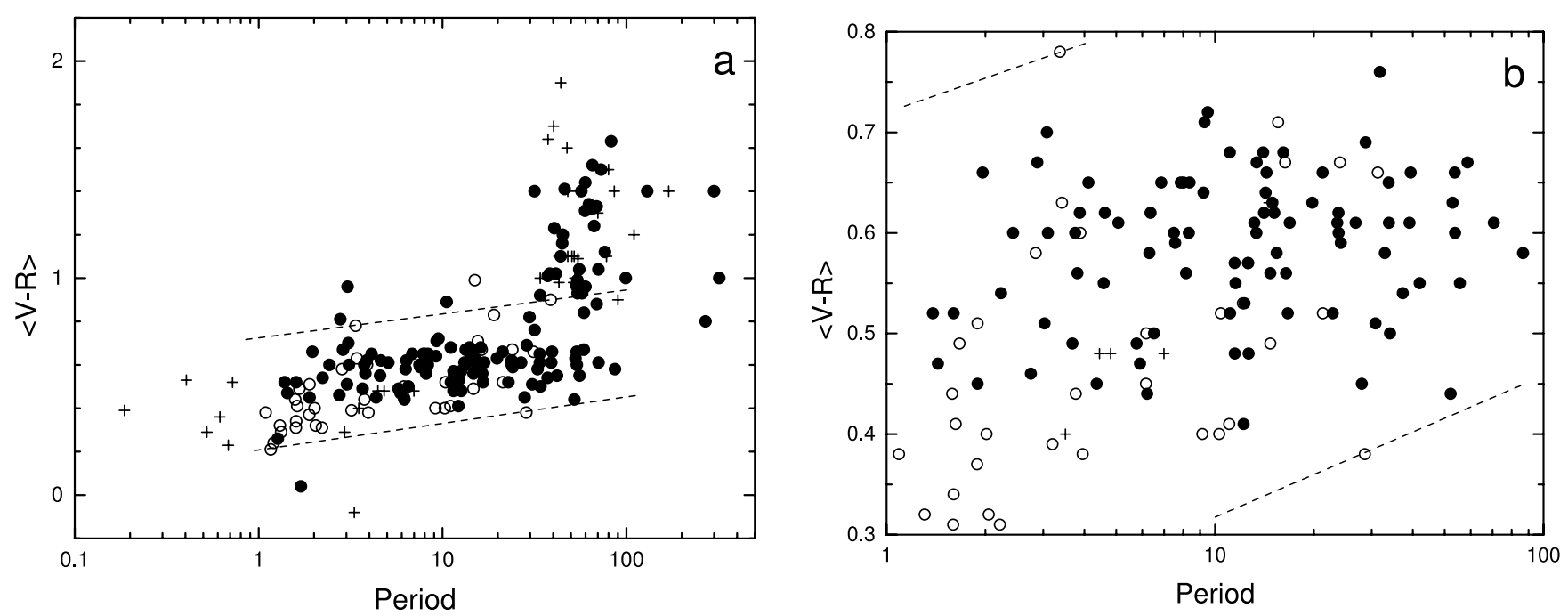

FIG. 2.-Mean colors plotted against period. All stars with periods in col. (2) of Table 3 are included. Crosses represent stars that were rejected on the basis of their light curves (CV, EC, IR, SP, or SL in col. [11] of Table 3). Filled circles represent the remaining stars from Table 3. Open circles represent known type II Cepheids from Schmidt et al. $(2004 \mathrm{a}, 2005 \mathrm{a}, 2005 \mathrm{~b})$. The dashed lines roughly delineate the region occupied by the open circles. Fig. $2 b$ is an enlargement of the central portion of Fig. $2 a$.

list no period or only list a timescale in Table 3 are too irregular to fit within the observed behavior of type II Cepheids. There are also stars that exhibit other irregularities (mainly in the NSVS data due to its temporal coverage) such as large light-curve changes from cycle to cycle, large changes in the mean brightness or amplitude or intervals of nonvariability. Such stars are rejected as Cepheid candidates and are flagged with IR in column (11) of Table 3.

Five stars showed no significant variation in our photometry. This is indicated by CO in column (11) of Table 3 . Without exception these stars had a companion of similar brightness within $1^{\prime}$. We conclude that the variation of the NSVS magnitudes is spurious and is caused by confusion between the two stars during the extraction of magnitudes.

Our revised periods for six stars were less than 0.75 day. These periods are too short to be of interest here and are flagged with SP in column (11).

The appearance of a number of light curves was obviously inappropriate for a Cepheid. These included apparent eclipsing binaries, denoted by EC in column (11), and a previously known cataclysmic variable, denoted by $\mathrm{CV}$.

The slope $S$, or, similarly, the comparison of the amplitudes in different bands has proven useful in distinguishing variations due to pulsation from those due to other causes, particularly eclipses (Schmidt et al. 1995; Schmidt \& Seth 1996; Jin et al. 2003). The small amplitudes of many of our stars introduce considerable uncertainty into the determination of $S$. Nonetheless, it is useful for eliminating some stars from consideration. Schmidt et al. (1995) found that pulsating stars had slopes in the range $0.18 \leq S \leq 0.24$. On the other hand, the bulk of the stars in Table 3 fall below this range with a median of $S=0.13$. This may be related to the small amplitudes in the present sample or to some other factor. Under these circumstances it is difficult to determine a lower limit for $S$ for pulsating stars. However, it is clear that pulsating stars cannot exhibit negative values of $S$. Accordingly, we have flagged all stars with $S$ more than two standard deviations below zero with SL in column (11) and do not consider them to be Cepheid candidates.

The mean colors, $\langle V-R\rangle$, are plotted against period in Figure 2 . Stars that were rejected as Cepheid candidates in the preceding paragraphs are plotted as crosses. The remainder are represented by filled circles. For comparison we have also plotted colors of known type II Cepheids from Schmidt et al. (2004a, 2005a, 2005b), referred to in those papers as high- $Z$ stars, as open circles. The dashed lines were drawn by eye to roughly delineate the expected region for type II Cepheids as defined by these stars.

Two stars fall below, i.e., to the blue of, the lower dashed line in Figure 2. These stars are rejected as Cepheids on this basis and are identified by the letters BL in column (11) of Table 3.

At periods longer than about 30 days a sequence of stars is apparent above (to the red of) the upper dashed line. Many of these show some irregularity in period or light-curve form. Most are likely to be long-period red variables. These stars are indicated by RE in column (11).

Finally, we note that a large portion of the stars from the present study form a band between $\langle V-R\rangle=0.42$ and 0.70 which extends from a period of 1.4 to 87 days. This band is entirely between the dashed lines and coincides with the locus of the majority of the open circles. Based on this, we regard the stars between the dashed lines that are represented by filled circles as the most promising Cepheid candidates. They are identified by an A in column (12) of Table 3.

Due to reddening some Cepheids may fall above the Cepheid sequence in Figure 2. Stars above the upper dashed line with periods less than 30 days are possibly reddened Cepheids and are flagged with B in column (12). On the other hand, stars in a

TABLE 4

Cepheid Candidates with Inconsistent Periods

\begin{tabular}{|c|c|c|}
\hline Star & $P_{\text {Beh }}$ & $P_{\mathrm{NSVS}}$ \\
\hline $\mathrm{R} 032 .$. & 1.6007 & 3.206 \\
\hline $\mathrm{R} 034 \ldots .$. & 3.68 & 3.65 \\
\hline $\mathrm{R} 043 \ldots \ldots \ldots$ & 5.078 & 5.118 \\
\hline $\mathrm{R} 053 \ldots \ldots$ & 2.23 & 7.36 \\
\hline $\mathrm{R} 063 \ldots \ldots$. & 3.811 & 11.0 \\
\hline 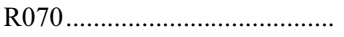 & 12.61 & 12.18 \\
\hline $\mathrm{R} 074 \ldots .$. & 23.8 & 25.7 \\
\hline $\mathrm{R} 080 \ldots \ldots \ldots$ & 2.140 & 7.11 \\
\hline R094 ............. & 9.3 & 24.5 \\
\hline R096........... & 11.1 & 23.2 \\
\hline R099............ & 55.5 & 35.2 \\
\hline 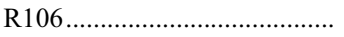 & 33.9 & 32.0 \\
\hline R131 & 55.7 & 55.1 \\
\hline 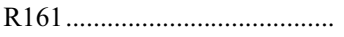 & 30.8 & 77 \\
\hline R165 & 69 & 50 \\
\hline
\end{tabular}



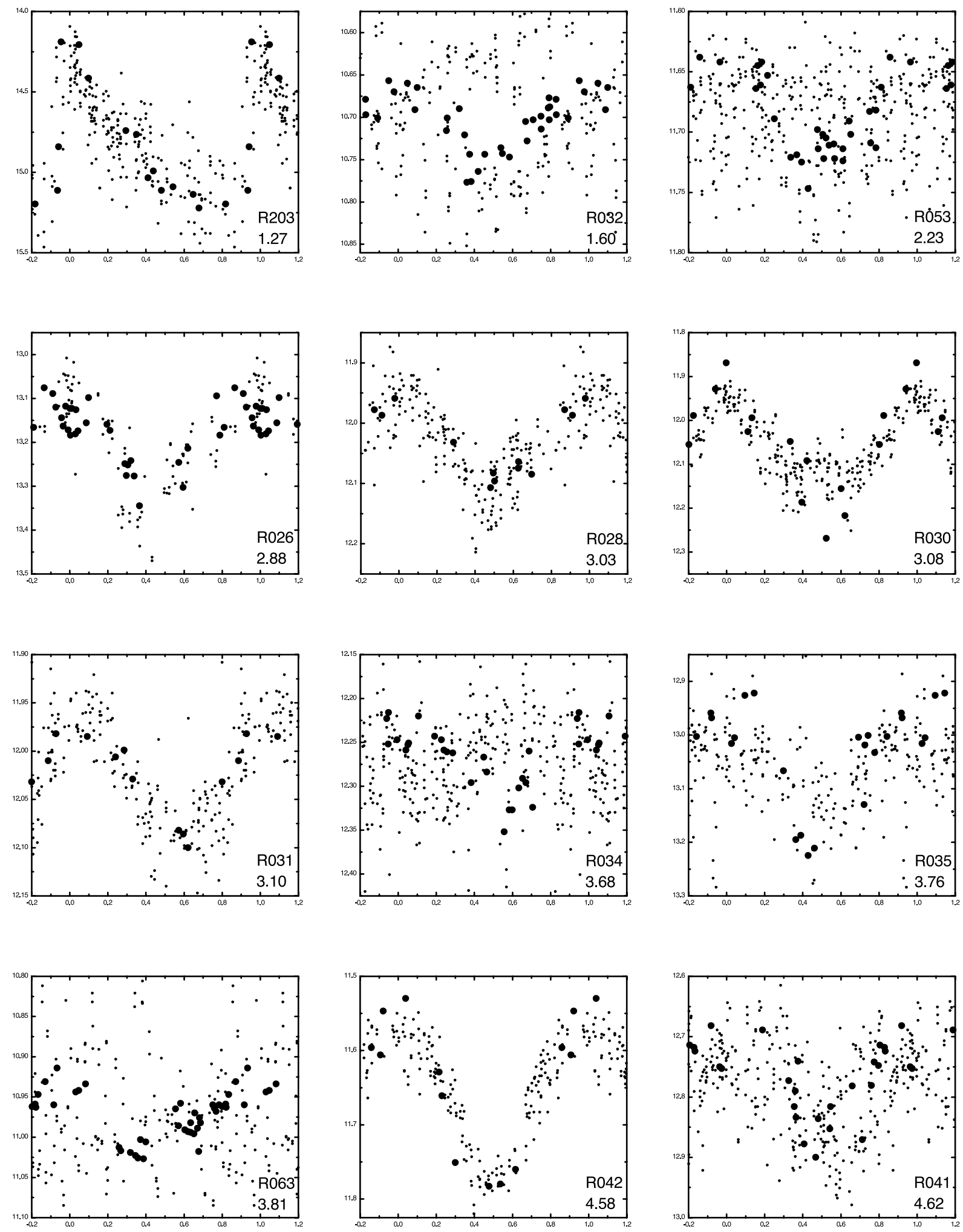

$\phi$

FIG. 3.- Light curves for all of the Cepheid candidates that were deemed to have nonsinusoidal light curves. Large symbols represent the present data, and small symbols represent data from the NSVS shifted in magnitude as described in the text. Relative phases were calculated using periods from Table 3, and the points were arbitrarily shifted in phase to place maximum light at approximately zero. 

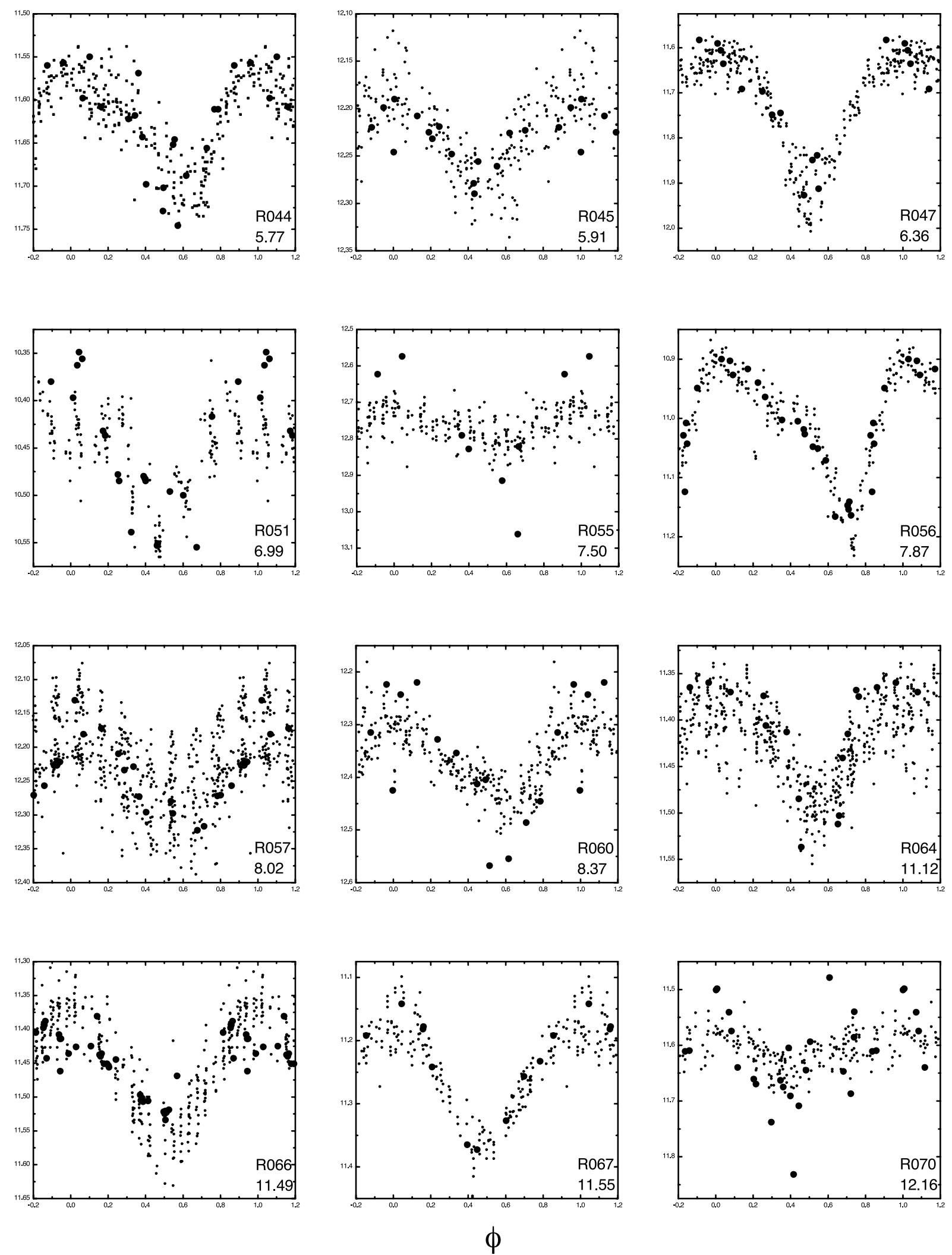

FIG. 3.-Continued 

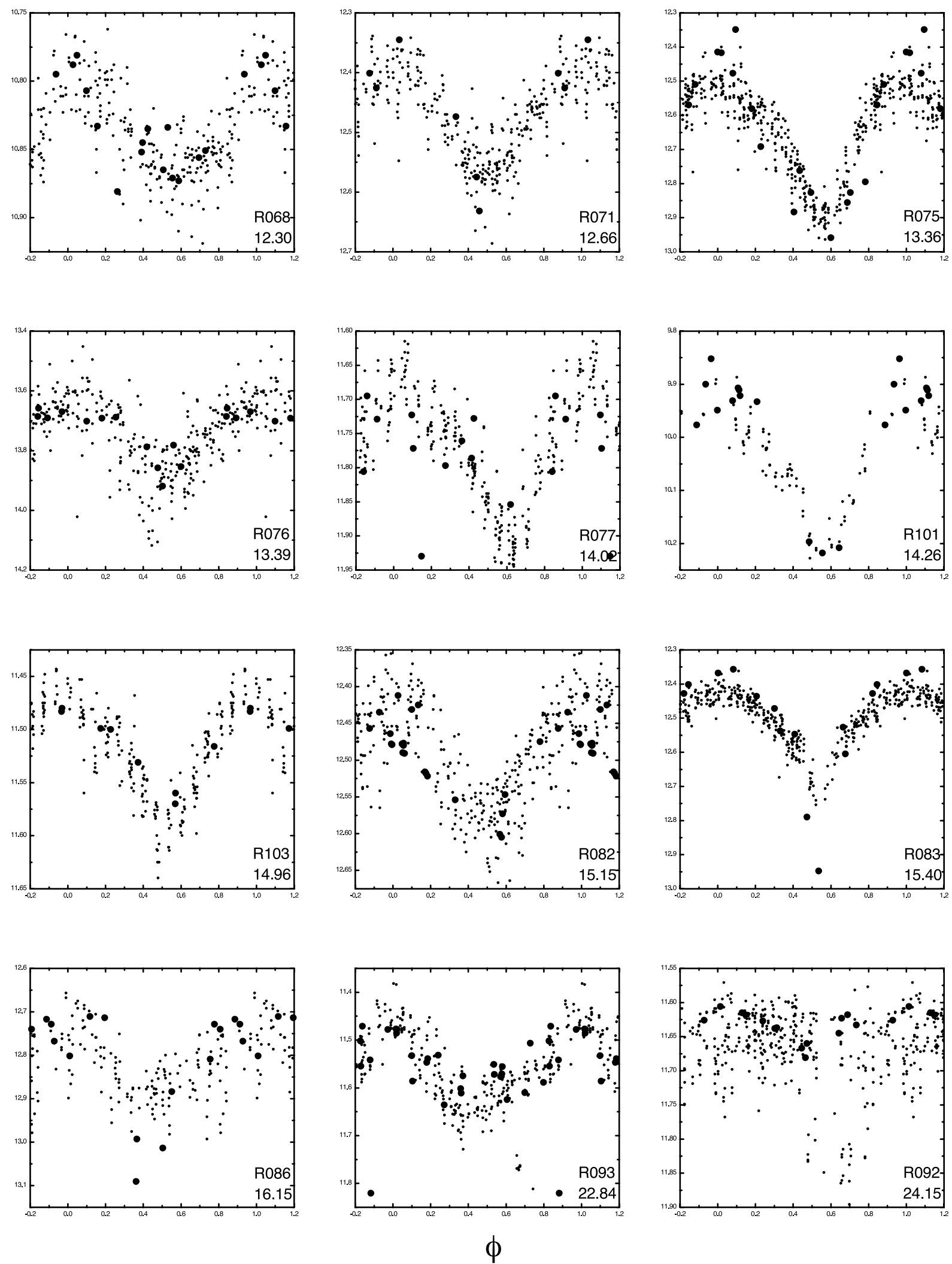

FIG. 3.-Continued 

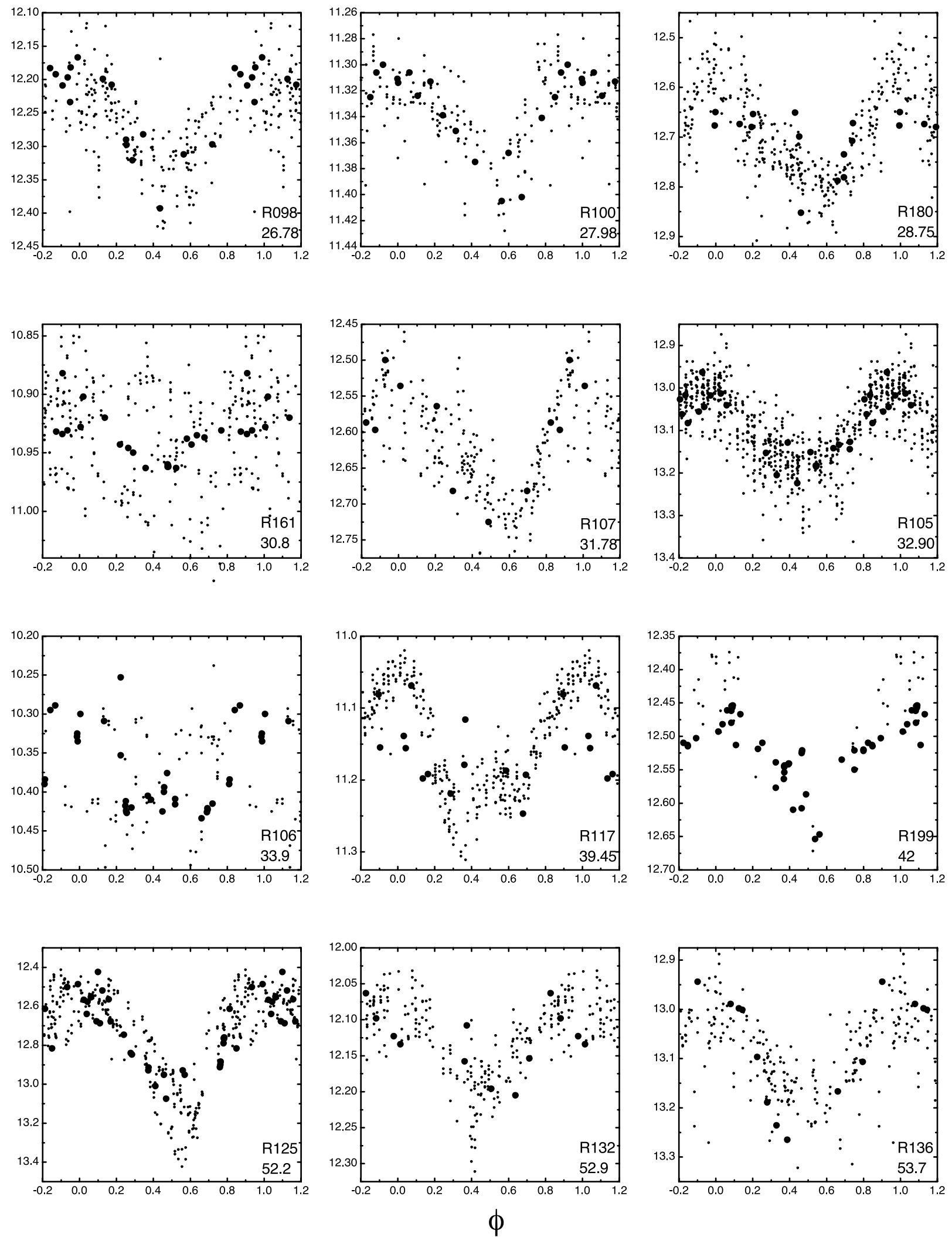

FIG. 3.-Continued 

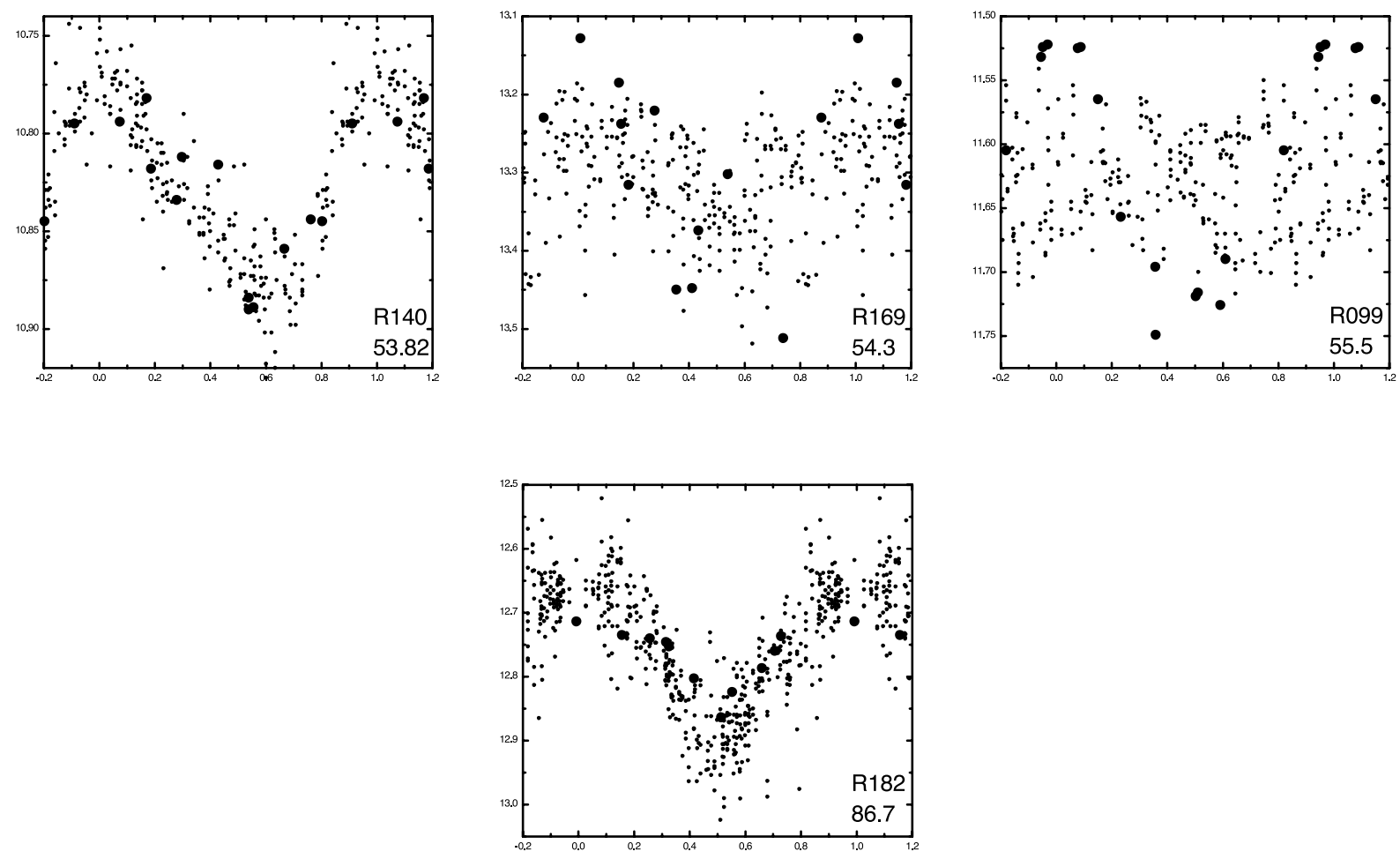

$\phi$

FIG. 3.-Continued

similar position at longer periods are probably a mixture of red variables and reddened Cepheids. Where we choose to separate the two groups is rather arbitrary; we have flagged such stars in the long-period range with $\langle V-R\rangle \leq 1.1$ as possible Cepheids (B in col. [12]). Again, these stars merit further observations to identify the Cepheids among them.

\section{DISCUSSION}

We only consider the Cepheid candidates (A or B in col. [12] of Table 3) in this section.

In Table 4 we have listed the Cepheid candidates for which we could not find a period that satisfied both the NSVS data and the present data (identified by footnote $b$ in Table 3 ). The second and third columns give the periods determined from our data and from the NSVS data. The period differences range from less than $1 \%$ to more than a factor of 3 . Even the smallest difference is more than an order of magnitude larger than those for known type II Cepheids (Schmidt et al. 2004a, 2005a, 2005b). We conclude that the differences between the second and third columns of Table 4 cannot be attributed to period changes of the type that are common in type II Cepheids.

Semiregular variables sometimes exhibit relatively sudden period changes that Cadmus et al. (1991) attributed to mode switching in at least one case. Kiss et al. (1999) found that about two-thirds of the 93 semiregular variables they studied were multiperiodic. The period ratios fell into five distinct groups and covered the range represented in Table 4. Buchler et al. (2004) later argued that this behavior was best explained by low-dimensional chaos. Since all of the stars studied by these authors had longer periods and nearly all were considerably redder than the stars in Table 4, it is not clear how relevant these results are to the present stars. However, chaos is of interest in connection with the pulsation of the type II Cepheids and their relationship to the RV Tau stars. Thus, we retain these stars as Cepheid candidates.

In Figure 3 we have plotted the light curves of selected Cepheid candidates. The periods from Table 3 were used to determine relative phases of the points, and each light curve was shifted by an arbitrary amount to place the maximum roughly at zero phase. As expected, the NSVS data show no periodicity for stars listed in Table 4.

For many of the stars, the scatter of the data points about a firstorder Fourier fit is no larger than that about a second or higher order fit. Thus, the data do not allow us to distinguish the light curve from a sinusoid. The plots are provided to allow the reader to examine the light-curve features discussed below. Since sinusoidal light curves are essentially featureless, they are not shown.

The first thing we note in examining the light curves is that many show significant scatter both in our photometry and in that from the NSVS. This is a common characteristic among type II Cepheids (Schmidt et al. 2004a, 2005a, 2005b), and the scatter in the present stars is quite comparable with the earlier observations of known type II Cepheids.

Considering only the present photometry (large circles in Fig. 3), the shapes of many of the light curves in Figure 3 resemble those of known type II Cepheids. Schmidt et al. (2004a) defined four types of light curves. Type A light curves were characterized by a rather flat maximum and a narrow, symmetric minimum near the middle of the cycle. Among the stars in Figure 3, R042, R047, R064, R067, R125, and R182 are clear examples of this morphology. Type B light curves exhibit a bump during declining light; R026, R056, and R066 are examples in the present sample. Type D light curves, similar to RRab stars, are exhibited by R030, $\mathrm{R} 107$, and R203. Although there are no type C light curves apparent among our stars, this could be because the form of the light 


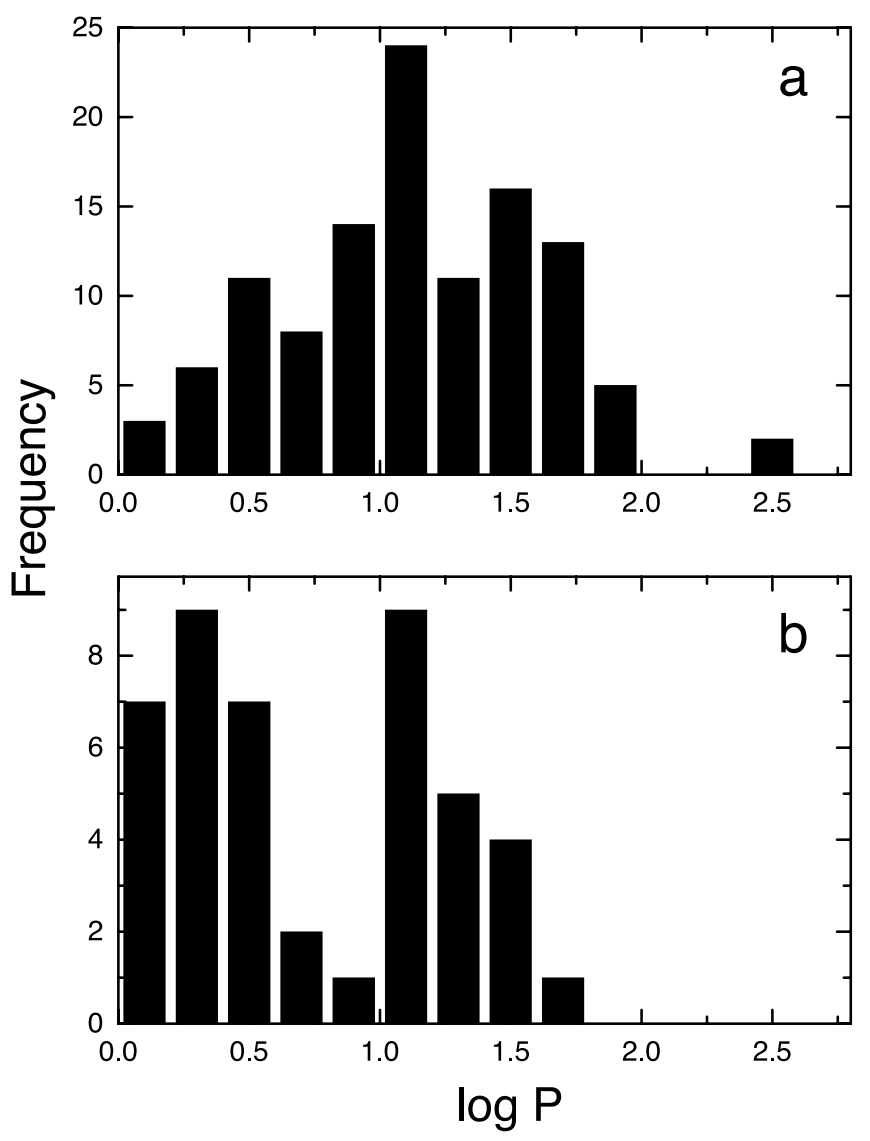

FIG. 4.-Distribution of periods of type II Cepheids. Panel $a$ shows the Cepheid candidates from the present sample (both status A and status B in col. [12] of Table 3 ), and panel $b$ shows previously observed stars.

curve is obscured by the small amplitude and scatter. We conclude that there is nothing in the appearance of the light curves in Figure 3 to eliminate any of them as Cepheid candidates.

We have plotted the amplitude distribution of the Cepheid candidates in Figure 1c. The smaller amplitudes, as compared to Figure $1 a$, are largely due to differences between the Akerlof et al. (2000) amplitudes and the $V$ amplitudes of individual stars rather than the selection of the Cepheid candidates. Comparing the distribution of the Cepheid candidate amplitudes with the known Cepheids (Fig. 1b) shows that the amplitude discrepancy noted in $\S 2$ has persisted; it is clearly intrinsic to the sample.

The period distribution for the Cepheid candidates is shown in Figure $4 a$. For comparison we show the period distribution for likely type II Cepheids from Schmidt et al. (2004a, 2005a, 2005b) in Figure $4 b$. It is obvious that the Cepheid candidates lack the gap between periods of 4 and 10 days $(0.6<\log P<1.0)$ and include fewer short-period stars than the likely type II Cepheids.

In Figure 5 we have plotted the $V$ amplitudes of the Cepheid candidates and known Cepheids against period. Since the amplitudes of all of these stars are based on the $V$ band and were determined in the same way, this comparison is more meaningful than that of Figure 1. It is noteworthy that five of the candidates

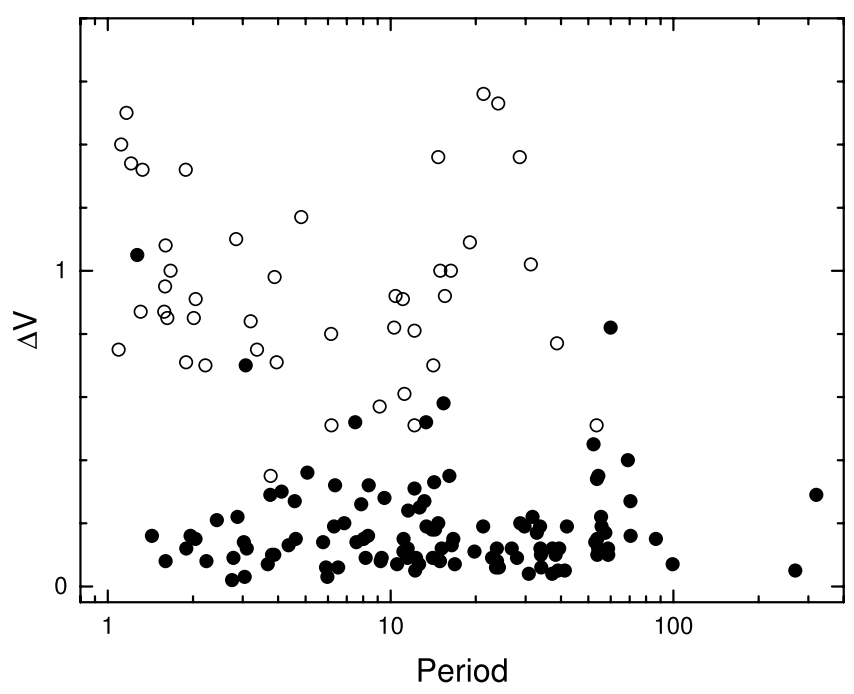

Fig. 5.- $V$ amplitude plotted against period. Filled circles represent Cepheid candidates from the present study, and open circles represent known type II Cepheids from Schmidt et al. (2004a, 2005a, 2005b).

fall among the known Cepheids, albeit toward the lower edge of the distribution. The remainder of the candidates exhibit no strong trend of amplitude with period except for a lack of amplitudes above 0.2 mag between 16 and 52 day periods and possibly shorter than 3 days. As we accumulate observations of a wider sample, the reality of these features will become clearer.

In summary, the various issues discussed above present a conundrum regarding the status of the Cepheid candidates. On one hand, they resemble known type II Cepheids in their location in the period-color diagram, the morphology of their light curves, the scatter in their light curves, and the stability of their pulsation between the NSVS observations and the present observations. On the other hand, the distributions of the amplitudes and periods are puzzling.

In a future paper we will analyze spectra of the Cepheid candidates. This will give us further insight into the nature of these stars and will help confirm or refute their identification as type II Cepheids.

This publication makes use of the data from the Northern Sky Variability Survey, created jointly by the Los Alamos National Laboratory and the University of Michigan. The NSVS was funded by the Department of Energy, the National Aeronautics and Space Administration, and the National Science Foundation. Equipment used in the observations was purchased with funds from NSF grant AST 00-97353. We are grateful to the Department of Physics and Astronomy at the University of Nebraska for continued support for the operation of Behlen Observatory. D. R. and L. T.-L. were supported during this work by the Undergraduate Creative Activities and Research Experiences program at the University of Nebraska. The authors are grateful to the referee, Christine Clement, for her thorough review of the manuscript and for her comments that helped to significantly improve it.
Akerlof, C., et al. 2000, AJ, 119, 1901

Bakos, G. A., Lazar, J., Papp, I., Sari, P., \& Green, E. M. 2002, PASP, 114, 974

Buchler, J. R., Kollath, Z., \& Cadmus, R. R., Jr. 2004, ApJ, 613, 532

Cadmus, R. R., Jr., Willson, L. A., Sneden, C., \& Mattei, J. A. 1991, AJ, 101, 1043

Chiosi, C. 1990, in Confrontation Between Stellar Pulsation and Evolution, ed.

C. Cacciari \& G. Clementini (San Francisco: ASP), 158

\section{REFERENCES}

Clement, C. M., et al. 2001, AJ, 122, 2587

Ferraz-Mello, S. 1981, AJ, 86, 619

Gingold, R. A. 1985, Mem. Soc. Astron. Italiana, 56, 169

Harris, H. C. 1985, AJ, 90, 756

Hartman, J. D., Bakos, G., Stanek, K. Z., \& Noyes, R. W. 2004, AJ, 128, 1761 
Jin, H., Kim, S.-L., Kwon, S.-G., Youn, J.-H., Lee, C.-U., Lee, D.-J., \& Kim, K.-S. 2003, A\&A, 404, 621

Kiss, L. L., Szatmary, K., Cadmus, R. R., \& Mattei, J. A. 1999, A\&A, 346, 542

Kovacs, G., \& Buchler, J. R. 1988, ApJ, 334, 971

Pojmanski, G., Pilecki, B., \& Szczygiel, D. 2005, Acta Astron., 55, 275

Schmidt, E. G., Chab, J. R., \& Reiswig, D. E. 1995, AJ, 109, 1239

Schmidt, E. G., Johnston, D., Langan, S., \& Lee, K. M. 2004a, AJ, 128, 1748 2005a, AJ, 129, 2007
Schmidt, E. G., Johnston, D., Langan, S., \& Lee, K. M. 2005b, AJ, 130, 832 Schmidt, E. G., Johnston, D., Lee, K. M., Langan, S., Newman, P. R., \& Snedden, S. A. 2004b, AJ, 128, 2988

Schmidt, E. G., Lee, K. M., Johnston, D., Newman, P. R., \& Snedden, S. A. 2003, AJ, 126, 906

Schmidt, E. G., \& Seth, A. 1996, AJ, 112, 2769

Wallerstein, G. 2002, PASP, 114, 689

Wozniak, P. R., et al. 2004, AJ, 127, 2436 